\title{
The Application of Dynamic Programming to Optimal Inventory Control
}

\author{
Daniel P. Berovic and Richard B. Vinter, Senior Member, IEEE
}

\begin{abstract}
This paper concerns a class of deterministic impulse control problems, arising in inventory control. A notable feature of the problem formulation is the presence of an end-point constraint. In consequence, the value function may be discontinuous. Viability theory provides a characterization of the value function as the unique lower semicontinuous solution to a Bensoussan-Lions type quasi-variational inequality (QVI), suitably interpreted for nondifferentiable, extended valued functions. Yet there are few examples in the literature of the use of this analytical machinery. This paper provides such an example. The example, which concerns a problem for which the value function is neither everywhere finite valued nor continuously differentiable on the interior of its effective domain, illustrates what is involved in calculating subdifferentials and checking satisfaction of QVI (in a generalized sense). This paper also provides a summary of the underlying theory, and gathers in the Appendix proofs of key results.
\end{abstract}

Index Terms-Dynamic programming, hybrid control, impulse control, operations research, optimal control.

\section{INTRODUCTION}

$\mathbf{T}$ HIS paper concerns the following "generalized inventory" or "impulse control" problem:

$$
\left(P_{0, x_{0}}\right)\left\{\begin{array}{l}
\text { Minimize } \int_{0}^{T} L(t, x(t), u(t)) d t+g(x(T))+C(v) \\
\text { subject to } \\
\frac{d x(t)}{d t}=f(t, x(t), u(t))+\frac{d v(t)}{d t} \quad \text { a.e. } \quad t \in[0, T] \\
u(t) \in \Omega \text { a.e. } \quad t \in[0, T] \\
x\left(t_{0}\right)=x_{0}
\end{array}\right.
$$

the data for which comprise a nonnegative number $T$, functions $L: \Re \times \Re^{n} \times \Re^{m} \rightarrow \Re, f: \Re \times \Re^{n} \times \Re^{m} \rightarrow \Re^{n}$ and $d: \Re^{n} \rightarrow \Re$, an extended valued function $g: \Re^{n} \rightarrow \Re \cup\{+\infty\}$, sets $\Omega \subset \Re^{m}$ and $\mathcal{D} \subset \Re^{n}$ and a point $x_{0} \in \Re^{n}$. (The term $C(v)$ in the cost, which depends on $d$ and $\mathcal{D}$ will be explained presently.)

A control policy $(u, v)$ on the interval $[a, b] \subset[0, T]$ comprises a measurable function $u:[a, b] \rightarrow \Re^{m}$ satisfying

$$
u(t) \in \Omega \quad \text { a.e. } \quad t \in[a, b]
$$

Manuscript received September 27, 2002; revised November 2, 2003. Recommended by Associate Editor H. Zhang and Editor-in-Chief C. G. Cassandras. This work was supported in part by the Engineering and Physical Sciences Research Council of the U.K.

The authors are with the Department of Electrical and Electronic Engineering and the Centre for Process Systems, Imperial College of Science, Technology, and Medicine, London SW7 2BT, U.K. (e-mail: d.berovic@ic.ac.ik; r.vinter@ic.ac.uk).

Digital Object Identifier 10.1109/TAC.2004.826719 and an impulse control $v$

$$
v=\left\{t_{1}, \ldots, t_{N(v)}, \xi_{1}, \ldots, \xi_{N(v)}\right\}
$$

described by the number of impulses, a nonnegative integer $N(v)$, the impulse times $t_{1}, \ldots, t_{N(v)}$ which are real numbers such that $a \leq t_{1} \leq \cdots \leq t_{N(v)} \leq b$, and the impulses $\xi_{1}, \ldots, \xi_{N(v)}$ at these times, which are vectors in $\Re^{n}$ such that $\xi_{i} \in \mathcal{D}$ for $i=1, \ldots, N(v)$. Notice that we allow jump times to be coincident, since it may be favorable to execute a large jump, which can be implemented as a sum of jumps in the jump set $\mathcal{D}$.

We define a state trajectory corresponding to $(u, v)$ to be a piecewise Lipschitz continuous function $y:[a, b] \rightarrow \Re^{n}$, continuous from the right on $(a, b)$, satisfying

$$
\dot{x}(t)=f(t, x(t), u(t)) \quad \text { a.e. } \quad t \in[a, b]
$$

whose points of discontinuity are contained in the set $\left\{t_{1}, \ldots, t_{N(v)}\right\}$ and are such that

$$
x\left(t^{+}\right)-x\left(t^{-}\right)=\sum_{\left\{j: t_{j}=t\right\}} \xi_{j} .
$$

for all $t \in\left\{t_{1}, \ldots, t_{N(v)}\right\}$. Here, $x\left(t^{+}\right)$and $x\left(t^{-}\right)$denote the limit from the right and left, respectively. (We interpret $t^{-}=a$ if $t=0$ and $t^{+}=b$ if $t=b$.) A process $(y, u, v)$ on $[a, b]$ comprises a control policy $(u, v)$ on $[a, b]$ and an associated state trajectory $y$. The underlying time interval, for a control policy, state trajectory, etc., is taken to be $[0, T]$, if not otherwise specified.

Accordingly, the evolution of the state between jump times is governed by a differential equation with conventional control term $u$, which we are free to choose. The evolution of the system can also be controlled, however, by applying a finite number of impulses over the relevant time interval, each of which causes a jump in the state variable.

The optimal control problem is to minimize the cost function

$$
J_{0, x_{0}}(u, v)=\int_{0}^{T} L(t, x(t), u(t)) d t+g(x(T))+C(v)
$$

over control policies $(u, v)$. Here, $x$ is the state trajectory corresponding to $(u, v)$, for which $x(0)=x_{0}$. The term $C(v)$ on the right side denotes the "cost" of the impulse control, namely

$$
C(v)=\sum_{i=1}^{N(v)} d\left(\xi_{i}\right)
$$

A control policy for $\left(P_{0, t}\right)$ which minimizes the cost is called an optimal control policy. An associated process is called an optimal process. 
A special case of the class of problems studied here is the inventory problem

$$
\left(Q_{0, x_{0}}\right)\left\{\begin{array}{l}
\text { Minimize } \int_{0}^{T} \tilde{L}(t, x(t)) d t+N(v) \\
\text { subject to } \\
\frac{d x(t)}{d t}=\tilde{f}(t, x(t))+\frac{d v(t)}{d t} \\
x(0)=x_{0}
\end{array}\right.
$$

investigated by Aubin [1], in which the differential equation $\dot{x}=\tilde{f}(t, x)$ describes the evolution of a vector valued stock. Impulse control action can be applied instantly to replenish stock at discrete times. In this case, the jump set $\mathcal{D}$ is taken to be $\mathcal{D}=\Re_{+}^{n}$ (the positive orthant of $\Re^{n}$ ). Control action incurs a unit transaction cost which is independent of the size of the stock increase. The cost function is chosen to penalize deviations from the vector of desired stock levels over the time horizon $[0, T]$. $\left(P_{0, x_{0}}\right)$ is a more general problem formulation than $\left(Q_{0, x_{0}}\right)$, because it allows for the action of conventional controls, a terminal cost and also a constraint on stock levels at the final time, namely

$$
x(T) \in\left\{x^{\prime} \in \Re^{n}: g\left(x^{\prime}\right)<+\infty\right\} .
$$

There is a substantial literature on deterministic and stochastic formulations of the inventory problem. Key early advances, most notably research establishing the link between value functions and quasivariational inequalities, were due to Bensoussan and Lions [7], [16]. Subsequent research in a deterministic framework allowed for both 'ordinary' and impulse control action, as in this paper; the principal goal was on characterizing value functions as unique continuous, uniformly bounded viscosity solutions of Bensoussan-Lions type quasi-variational inequalities (QVIs) [5], [15], and [9]. Reference [8] provides a general framework for studying hybrid control systems, numerous references to the early literature and an expository account of the role of viscosity solutions in the analysis of the value function for optimal hybrid control problems. For the most part, these references concern infinite time horizon problems, with discounted cost, not the finite time interval problem of this paper.

A distinctive feature of our formulation of the generalized inventory problem is that it allows an endpoint constraint on state trajectories. In consequence, we can expect the value function to be discontinuous and even to have domain a strict subset of the "initial conditions" space. As shown in [12], viability theory can be used to link value functions and generalized lower semicontinuous solutions to QVI, even in this general setting. (The link, in a viscosity solutions framework, was established by Barron and Jensen [6].) General analytical tools for this purpose have been developed by Aubin and his co-workers. See [3], which explores implications of viability theory regarding properties of hybrid systems (including inventory models) and [4] which focuses on characterization of lower semicontinuous value functions for infinite horizon, discounted cost impulse control problems. A recent paper, using viability theory to provide information about the domain for certain optimal hybrid control problems with state constraints is [11].

While there have been striking advances in the theory relating to the description of lower semicontinuous value functions as unique generalized solutions to QVIs, there are available in the literature few examples of solutions to specific inventory problems, illustrating the use of this analytic machinery. The main purpose of this paper is to provide such an example. We note that, here, the value function is extended valued and fails to be continuously differentiable on the interior of its effective domain. Our analysis provides a candidate for the value function, and confirms that it is so by showing that it is a generalized, lower semicontinuous solution to QVI. Our example, which illustrates the steps involved in calculating subdifferentials and verifying satisfaction of QVI in one case, is intended to serve as a template for determining value functions in more complicated situations. The paper begins with a summary of relevant theory. Proofs underlying the main steps are gathered in the Appendix.

\section{Characterization of The VAlue Function}

Consider the generalized inventory problem $\left(P_{0, x_{0}}\right)$ of the introduction. We will invoke the following hypotheses, in which $\tilde{f}(t, x, u)=(f(t, x, u), L(t, x, u))$.

H1) $\Omega$ is compact set.

H2) $\tilde{f}$ is continuous and there exists $k>0, c>0$ such that

$$
\left|\tilde{f}(t, x, u)-\tilde{f}\left(t, x^{\prime}, u\right)\right| \leq k\left|x-x^{\prime}\right| \quad|\tilde{f}(t, x, u)| \leq c
$$

for all $t \in[0, T], x, x^{\prime} \in \Re^{n}, u \in \Omega$.

H3) $L$ is bounded below on $[0, T] \times \Re^{n} \times R^{m}$ and, for each $(t, x) \in \Re \times \Re^{n}$, the set

$$
\begin{aligned}
& \left\{(v, \alpha) \in \Re^{n} \times \Re: v=f(t, x, u),\right. \\
& \quad \alpha \geq L(t, x, u) \text { for some } u \in \Omega\}
\end{aligned}
$$

is convex.

H4) $d$ is continuous and there exists $r>0$ such that $d(x)>$ $r$ for all $x \in \Re^{n}$.

H5) $g$ is a lower semicontinuous function that is bounded below and satisfies

$$
g(x) \leq \min _{\xi \in \mathcal{D}}\{g(x+\xi)+d(\xi)\} \quad \text { for all } x \in \Re^{n} .
$$

H6) $\mathcal{D}$ is compact.

Comments on Hypotheses: H1) and H2) ensure that, corresponding to any control policy and initial state, there exists a unique state trajectory. H3), H4), and H6), together with the condition that $g$ is lower semicontinuous and bounded below, ensure the existence of a minimizer for $\left(P_{0, x_{0}}\right)$. The role of H5) is to exclude the possibility of a jump in the optimal policy at the final time.

Embed $\left(P_{0, x_{0}}\right)$ in the family of problems $\left\{\left(P_{t, x}\right):(t, x) \in\right.$ $\left.[0, T] \times \Re^{n}\right\}$. Here, $\left(P_{t, x}\right)$ denotes a modified version of $\left(P_{0, x_{0}}\right)$, in which the initial data $(t, x)$ replaces $\left(0, x_{0}\right)$.

The value function $V:[0, T] \times \Re^{n} \rightarrow \Re \cup+\{\infty\}$ is

$$
V(t, x)=\inf \left(P_{t, x}\right), \quad(t, x) \in[0, T] \times \Re^{n} .
$$

(The right side denotes "infimum cost of $\left(P_{t, x}\right)$ ".)

Define the Hamiltonian function $\mathcal{H}:[0, T] \times \Re^{n} \times \Re^{n}$

$$
\mathcal{H}(t, x, p)=\max _{u \in \Omega}\{p \cdot f(t, x, u)-L(t, x, u)\} .
$$


The value function is linked with solutions, in some appropriate sense, to a Bensoussan-Lions type QVI for problem $\left(P_{0, x_{0}}\right)$, namely functions $\phi$ satisfying the following conditions:

$$
\begin{aligned}
& \phi_{t}-\mathcal{H}\left(t, x,-\phi_{x}\right) \geq 0 \\
& \quad \text { for all }(t, x) \in(0, T) \times \Re^{n} \\
& \phi(t, x) \leq \min _{\xi \in \mathcal{D}}\{\phi(t, x+\xi)+d(\xi)\} \\
& \quad \text { for all }(t, x) \in[0, T] \times \Re^{n} \\
& \left(\phi_{t}-\mathcal{H}\left(t, x,-\phi_{x}\right)\right) \cdot\left(\phi-\min _{\xi \in \mathcal{D}}\{\phi+d\}\right)=0 \\
& \quad \text { for all }(t, x) \in(0, T) \times \Re^{n} \\
& \phi(T, x)=g(x) \quad \text { for all } x \in \Re^{n} .
\end{aligned}
$$

Under hypotheses $\mathrm{H} 1$ )-H6), the value function may fail to be continuously differentiable. The value function is lower semicontinuous in these circumstances, however. To exploit this fact, it is necessary to interpret lower semicontinuous functions that are said to satisfy QVI.

There are a number of ways to do this. Our interpretation is based on the notion of the proximal subdifferential, whose properties are developed in, for example, [10] or [13]. Let $\mathcal{B}$ denote the closed unit ball. Given an open set $\mathcal{O} \subset \Re^{k}$, a function $\psi: \mathcal{O} \rightarrow \Re \cup\{+\infty\}$ and a point $\bar{y} \in \mathcal{O}$ such that $\psi(\bar{y})<+\infty$, the proximal subdifferential of $\psi$ at $\bar{y}$, written $\partial^{P} \psi(\bar{y})$, is

$\partial^{P} \psi(\bar{y}):=\left\{\eta \in \Re^{k}:\right.$ there exists $M>0, \epsilon>0$ such that

$$
\left.\psi(y)-\psi(\bar{y}) \geq \eta \cdot(y-\bar{y})-M|y-\bar{y}|^{2} \quad \forall y \in \bar{y}+\epsilon \mathcal{B} .\right\} .
$$

Definition 2.1: A function $\phi:[0, T] \times \Re^{n} \rightarrow \Re \cup\{+\infty\}$ is said to be a lower semicontinuous solution of QVI, if it is lower semicontinuous and bounded below, and the following conditions are satisfied.

a) For each $(t, x) \in(0, T) \times \Re^{n}$

$$
\xi_{0}-\mathcal{H}\left(t, x,-\xi_{1}\right) \geq 0 \quad \forall\left(\xi_{0}, \xi_{1}\right) \in \partial^{P} \phi(t, x) .
$$

b) For each $(t, x) \in[0, T] \times \Re^{n}$ such that $\phi(t, x)<+\infty$,

$$
\phi(t, x) \leq \min _{\xi \in \mathcal{D}}\{\phi(t, x+\xi)+d(\xi)\} .
$$

c) For each $(t, x) \in(0, T) \times \Re^{n}$ such that $\phi(t, x)<+\infty$ and

$$
\phi(t, x)<\min _{\xi \in \mathcal{D}}\{\phi(t, x+\xi)+d(\xi)\}
$$

we have

$$
\xi_{0}-\mathcal{H}\left(t, x,-\xi_{1}\right)=0 \quad \forall\left(\xi_{0}, \xi_{1}\right) \in \partial^{P} \phi(t, x) .
$$

d) For each $x \in \Re^{n}$

$$
\liminf _{t^{\prime} \uparrow T, x^{\prime} \rightarrow x} \phi\left(t^{\prime}, x^{\prime}\right)=\phi(T, x)=g(x) .
$$

e) For each $x \in \Re^{n}$ such that $\phi(t, x)<+\infty$ and

$$
\phi(0, x)<\min _{\xi \in \mathcal{D}}\{\phi(0, x+\xi)+d(\xi)\}
$$

we have

$$
\phi(0, x)=\liminf _{t^{\prime} \downarrow 0, x^{\prime} \rightarrow x} \phi\left(t^{\prime}, x^{\prime}\right) .
$$

Notice that, when $\phi$ is of class $C^{1}$, then conditions a)-e) imply conditions (2.2)-(2.5). Thus, lower semicontinuous solutions, as we define them, reduce to classical-sense solutions for sufficiently regular functions. (We use here the fact that, for a $C^{1}$ function $\psi$, we have $\partial^{P} \psi(\bar{y})=\{\nabla \psi(\bar{y})\}$.) Concerning links with viscosity solutions, we note, in particular, that assessing whether condition a) of the previous definition is satisfied involves checking the relevant inequality when $\left(\xi_{0}, \xi_{1}\right)$ is the gradient of quadratic "test function" that minorizes the Hamiltonian at the basepoint $(t, x)$. Thus, the condition is related to (but somewhat less restrictive) than the condition that $\phi$ is a viscosity subsolution, where the inequality is required to hold for the larger class of $C^{1}$ test functions. It can be shown likewise (see [10]) that the property that $\phi$ is a lower semicontinuous viscosity solution to the Hamilton Jacobi equation in the sense of [6], at relevant points in its domain, is a sufficient condition that condition c) is satisfied in the sense of the previous definition.

The following theorem gives conditions under which the set of lower semicontinuous solutions to QVI precisely captures the value function for problem $\left(P_{0, x_{0}}\right)$.

Theorem 2.2: Assume $\mathrm{H} 1)-\mathrm{H} 6)$. Then, the value function $V:[0, T] \times \Re^{n} \rightarrow \Re \cup\{+\infty\}$ is the unique lower semicontinuous function to QVI.

Theorem 2.2 provides a necessary and sufficient condition for a lower semicontinuous function to be the value function: It must be a lower semicontinuous solution to QVI. If we are content with merely a sufficient condition along these lines, namely a "verification theorem," we can relax the hypotheses (dropping the requirement that $\mathcal{D}$ is compact) and state the condition in terms of a function satisfying just some of the defining conditions of lower semicontinuous solutions to QVI.

Proposition 2.3: Assume H1)-H5). Let $(y, u, v)$ be a process for $\left(P_{0, x_{0}}\right)$. Suppose there exists a lower semicontinuous function $\phi:[0, T] \times \Re^{n} \rightarrow \Re \cup\{+\infty\}$ such that

$$
\left.\mathrm{a}^{\prime}\right) \text { for each }(t, x) \in(0, T) \times \Re^{n}
$$

$$
\xi_{0}-\mathcal{H}\left(t, x,-\xi_{1}\right) \geq 0 \quad \forall\left(\xi_{0}, \xi_{1}\right) \in \partial^{P} \phi(t, x) ;
$$

$\left.\mathrm{b}^{\prime}\right)$ for each $(t, x) \in[0, T] \times \Re^{n}$ such that $\phi(t, x)<+\infty$

$$
\phi(t, x) \leq \min _{\xi \in \mathcal{D}}\{\phi(t, x+\xi)+d(\xi)\} ;
$$

$\left.\mathrm{d}^{\prime}\right)$ for each $x \in \Re^{n}$

$$
\liminf _{t^{\prime} \uparrow T, x^{\prime} \rightarrow x} \phi\left(t^{\prime}, x^{\prime}\right)=\phi(T, x)=g(x) .
$$

Assume, furthermore, that

$$
\phi\left(0, x_{0}\right)=\int_{0}^{T} L(t, y(t), u(t)) d t+g(y(T))+C(v) .
$$

Then, $(y, u, v)$ is an optimal process, and $\phi\left(0, x_{0}\right)$ is the minimum cost for $\left(P_{0, x_{0}}\right)$.

\section{Construction of Optimal Policies}

The preceding theorem tells us that, in principle, we can obtain the value function by finding a lower semicontinuous solution $\phi$ to QVI. Of course, of primary interest is the optimal control policy (for a specified initial state). In this section, we 
address the problem of deriving an optimal control policy from the value function.

It is convenient to introduce a new representation of a process $(y, u, v)$ on some subinterval $[a, b] \subset[0, T]$. Here attention focuses on the time intervals between impulse times, rather than the impulse times themselves.

We say that a family of elements $\left\{\left[t_{i}^{-}, t_{i}^{+}\right],\left(x_{i}, u_{i}\right)\right\}_{i=0}^{N}$ provides a multiprocess representation of a process $\left(y, u, v=\left(t_{1}, \ldots, t_{N}, \xi_{1}, \ldots, \xi_{N}\right)\right)$ on $[a, b]$ if

i) $t_{0}^{-}=a, t_{N}^{+}=b$ and $t_{i}=t_{i-1}^{+}=t_{i}^{-}$for $i=1, \ldots, N$;

ii) $\left(x_{i}, u_{i}\right)$ is an impulse-free process on $\left[t_{i}^{-}, t_{i}^{+}\right]$for $i=$ $0, \ldots, N$

iii) $x_{i}\left(t_{i}^{-}\right)-x_{i-1}\left(t_{i-1}^{+}\right)=\xi_{i}, i=1, \ldots, N$;

iv) $u(t)=\sum_{i=0}^{N} \chi_{\left[t_{i}^{-}, t_{i}^{+}\right)}(t) u_{i}(t)$ a.e., and

$$
y(t)= \begin{cases}x_{1}\left(t_{0}^{-}\right), & \text {if } t=a \\ \sum_{i=0}^{N} \chi_{\left[t_{i}^{-}, t_{i}^{+}\right)}(t) x_{i}(t), & \text { if } t \in(a, b) \\ x_{N}\left(t_{N}^{+}\right), & \text {if } t=b .\end{cases}
$$

Here, $\chi_{A}$ is the function

$$
\chi_{A}(z)= \begin{cases}1, & \text { if } z \in A \\ 0, & \text { otherwise. }\end{cases}
$$

It is a straightforward matter to show that every set of elements $\left\{\left[t_{i}^{-}, t_{i}^{+}\right],\left(u_{i}, x_{i}\right)\right\}_{i=0}^{N}$ satisfying i)-iv) defines a process $\left(y, u, v=\left(t_{1}^{+}, \ldots, t_{N}^{+}, \xi_{1}, \ldots, \xi_{N}\right)\right)$ on $[a, b]$ and, conversely, every process on $[a, b]$ has a multiprocess representation.

For a lower semicontinuous function $\phi:[0, T] \times \Re^{n} \rightarrow \Re \cup$ $\{+\infty\}$ we define the function $R_{\phi}:[0, T] \times \Re^{n} \rightarrow \Re \cup\{+\infty\}$ $R_{\phi}(t, x)$

$$
:= \begin{cases}\phi(t, x)-\min _{\xi \in \mathcal{D}}\{\phi(t, x+\xi)+d(\xi)\}, & \text { if } \phi(t, x)<\infty \\ +\infty, & \text { otherwise. }\end{cases}
$$

Recall that, if $\phi$ is a lower semicontinuous solution of QVI, then $R_{\phi}(t, x) \leq 0$ for all $(t, x) \in[0, T] \times \Re^{n}$ such that $\phi(t, x)<$ $+\infty$. It is also relevant to the constructions below that, if $\phi$ is a lower semicontinuous solution to QVI and $\left(t^{-}, x^{-}\right)$is a point in $[0, T) \times \Re^{n}$ such that

$$
R_{\phi}\left(t^{-}, x^{-}\right)<0
$$

then there always exists $t^{+} \in\left(t^{-}, T\right]$ and an impulse-free process $(x, u)$ on $\left[t^{-}, t^{+}\right]$such that $x\left(t^{-}\right)=x_{0}$

$$
V\left(t^{+}, x\left(t^{+}\right)\right)=-\int_{t^{-}}^{t^{+}} L(t, x(t), u(t)) d t+V\left(t^{-}, x\left(t^{-}\right)\right)
$$

and either $t^{+}=T$ or $R_{\phi}\left(t^{+}, x\left(t^{+}\right)\right)=0$.

Algorithm 3.1: Data: A lower semicontinuous solution $\phi$ to QVI satisfying $\phi\left(0, x_{0}\right)<+\infty$.

Purpose: To construct a process $(x, u, v)$ for $\left(P_{0, x_{0}}\right)$, such that

$$
\phi\left(0, x_{0}\right)=\int_{0}^{T} L(t, x(t), u(t)) d t+g(x(T))+C(v) .
$$

(The following steps describe the multiprocess representation $\left\{\left[t_{i}^{-}, t_{i}^{+}\right],\left(x_{i}, u_{i}\right)\right\}_{i=0}^{N}$ of $(x, u, v)$, with jumps $\xi_{1}=x_{1}\left(t_{1}^{-}\right)-$ $x_{0}\left(t_{0}^{+}\right), \ldots, \xi_{N}=x_{N}\left(t_{N}^{-}\right)-x_{N-1}\left(t_{N-1}^{+}\right)$

Initialization: Set $t_{0}^{-}=0$.
If $R_{\phi}\left(t_{0}^{-}, x_{0}\right)=0$ set $t_{0}^{+}=t_{0}^{-}$and take $\left(x_{0}(),. u_{0}().\right)$ to be the trivial impulse-free process $\left(x_{0}(.) \equiv x_{0}, u_{0}(.) \equiv \bar{u}\right)$ for some arbitrary $\bar{u} \in \Omega$.

If $R_{\phi}\left(t_{0}^{-}, x_{0}\right)<0$, choose $t_{0}^{+} \in\left[t_{0}^{-}, T\right]$ and an impulse-free process $\left(x_{0}, u_{0}\right)$ on $\left[t_{0}^{-}, t_{0}^{+}\right]$such that

$V\left(t_{0}^{+}, x_{0}\left(t_{0}^{+}\right)\right)=-\int_{t_{0}^{-}}^{t_{0}^{+}} L\left(t, x_{0}(t), u_{0}(t)\right) d t+V\left(t_{0}^{-}, x_{0}\left(t_{0}^{-}\right)\right)$

and either $t_{0}^{+}=T$, or $R_{\phi}\left(t_{0}^{+}, x_{0}\left(t_{0}^{+}\right)\right)=0$.

If $t_{0}^{+}=T$, set $N=0$ and terminate the algorithm. Otherwise, we have $t_{0}^{+}<T$ and $R_{\phi}\left(t_{0}^{+}, x_{0}\left(t_{0}^{+}\right)\right)=0$, and it is possible to construct impulse-free processes $\left(x_{1}(),. u_{1}().\right), \ldots,\left(x_{N}(),. u_{N}().\right)$ on the contiguous intervals $\left[t_{1}^{-}, t_{1}^{+}\right], \ldots,\left[t_{N}^{-}, t_{N}^{+}\right]$, according to the following recursive procedure.

Recursion: Given an integer $i \geq 1$ and an impulse-free process $\left(x_{i-1}(),. u_{i-1}().\right)$ on $\left[t_{i-1}^{-}, t_{i-1}^{+}\right]$such that $t_{i-1}^{+}<T$ and $R_{\phi}\left(t_{i-1}^{+}, x_{i}\left(t_{i-1}^{+}\right)\right)=0$, we construct $\xi_{i} \in \mathcal{D}$ and an impulse-free process $\left(x_{i}(),. u_{i}().\right)$ on $\left[t_{i}^{-}, t_{i}^{+}\right]$such that $t_{i}^{-}=t_{i-1}^{+}, t_{i}^{+} \in\left[t_{i}^{-}, T\right]$ as follows.

Set $t_{i}^{-}=t_{i-1}^{+}$. Choose $\xi_{i}$ such that

$$
\phi\left(t_{i-1}^{+}, x_{i-1}\left(t_{i-1}^{+}\right)\right)=\phi\left(t_{i-1}^{+}, x_{i-1}\left(t_{i-1}^{+}\right)+\xi_{i}\right)+d\left(\xi_{i}\right) .
$$

If $R_{\phi}\left(t_{i-1}^{+}, x_{i}\left(t_{i-1}^{+}\right)+\xi_{i}\right)=0$ set $t_{i}^{+}=t_{i}^{-}$and take $\left(x_{i}(),. u_{i}().\right)$ to be the trivial process $\left(x_{i}(.) \equiv\right.$ $\left.x_{i-1}\left(t_{i-1}^{+}\right)+\xi_{i}, u_{i}(.) \equiv \bar{u}\right)$. We have $t_{i}^{+}<T$ and $R_{\phi}\left(t_{i}^{+}, x_{i}\left(t_{i}^{+}\right)\right)=0$.

If, on the other hand, $R_{\phi}\left(t_{i-1}^{+}, x_{i-1}\left(t_{i-1}^{+}\right)+\xi_{i}\right)<0$, then we choose $t_{i}^{+} \in\left(t_{i}^{-}, T\right]$ and an impulse-free process $\left(x_{i}(),. u_{i}().\right)$ on $\left[t_{i}^{-}, t_{i}^{+}\right]$such that $x_{i}\left(t_{i}^{-}\right)=x_{i-1}\left(t_{i-1}^{+}\right)+\xi_{i}$

$$
\phi\left(t_{i}^{+}, x_{i}\left(t_{i}^{+}\right)\right)=-\int_{t_{i}^{-}}^{t_{i}^{+}} L\left(t, x_{i}(t), u_{i}(t)\right) d t+\phi\left(t_{i}^{-}, x_{i}\left(t_{i}^{-}\right)\right)
$$

and either $t_{i}^{+}=T$ or $R_{\phi}\left(t_{i}^{+}, x_{i}\left(t_{i}^{+}\right)\right)=0$.

If $t_{i}^{+}=T$, set $N=i$ and terminate the algorithm.

(Note that if an application of the recursion does not terminate the algorithm, an impulse-free process $\left(x_{i+1}(),. u_{i+1}().\right)$ on $\left[t_{i+1}^{-}, t_{i+1}^{+}\right]$results, which satisfies the relevant conditions for a fresh application of the recursion, namely $t_{i+1}^{+}<T$ and $R_{\phi}\left(t_{i+1}^{+}, x_{i+1}\left(t_{i+1}^{+}\right)\right)=0$. $)$

Theorem 3.2: Assume H1)-H6). Algorithm 3.1 terminates after a finite number of recursions and yields a minimizing process for $\left(P_{0, x_{0}}\right)$.

The term "algorithm" here is something of a misnomer because, given a point $\left(t^{-}, x^{-}\right) \in[0, T) \times \Re^{n}$ such that $R\left(t^{-}, x^{-}\right)<0$, the procedure requires us to find a 'maximal' impulse-free process $(x(t), u(t))$ on $\left[t^{-}, t^{+}\right]$, with $x\left(t^{-}\right)=x^{-}$ and graph in

$$
\left\{(t, x) \in[0, T] \times \Re^{n}: R(t, x)<0\right\}
$$

and such that

$$
V\left(t^{+}, x\left(t^{+}\right)\right)=-\int_{t^{-}}^{t^{+}} L(t, x(t), u(t)) d t+V\left(t^{-}, x\left(t^{-}\right)\right) .
$$


While it is true that such a process exists, it is, in general, a nonconstructive step to find it. In certain cases of interest, this step is constructive, however. The simplest is that when neither $f$ nor $L$ depend on $u$. (Write $f(t, x, u)=f^{\prime}(t, x)$ ).) In this situation, there is a unique such process obtained by solving the differential equation

$$
\begin{aligned}
\dot{x} & =f^{\prime}(t, x(t)) \quad \text { a.e. } t \in\left[t^{-}, t^{+}\right] \\
x\left(t^{-}\right) & =x^{-}
\end{aligned}
$$

where $t^{+}$is the first exit time of $(t, x(t))$ from the region (3.1) (or $t^{+}=T$, if we remain within this region). Here, the algorithm tells us to solve the differential equation forward in time, for initial data $\left(t^{-}, x^{-}\right)$, until the end time is reached $\left(t^{+}=T\right)$, or until $R_{\phi}\left(t^{+}, x\left(t^{+}\right)\right)=0$. In the latter case, we jump a distance $\xi$, where $\xi \in \mathcal{D}$ is chosen to satisfy

$$
\phi\left(t^{+}, x\left(t^{+}\right)\right)=\phi\left(t^{+}, x\left(t^{+}\right)+\xi\right)+d(\xi)
$$

and so on.

\section{AN EXAMPLE}

In this section, we illustrate the application of the preceding theory by using it to solve a special case of the inventory problem, namely

$$
\left(E_{0, x_{0}}\right)\left\{\begin{array}{l}
\text { Minimize } \int_{0}^{T}|x(t)| d t+N(v) \\
\text { subject to } \\
\frac{d x(t)}{d t}=-1+\frac{d v(t)}{d t} \\
x(0)=x_{0} \text { and } x(T) \leq 0 .
\end{array}\right.
$$

Here, a control policy $v$ is an impulse control

$$
v=\left\{t_{1}, \ldots, t_{N(v)}, \xi_{1}, \ldots, \xi_{N(v)}\right\}
$$

giving rise to jumps $\xi_{1}, \ldots, \xi_{N(v)}$ in the state trajectory at times $t_{1}, \ldots, t_{N(v)}$, respectively. The jumps are required to satisfy

$$
\xi_{i} \geq 0, \quad \text { for } i=1, \ldots, N(v) .
$$

There is no conventional control component. In the present context, we omit reference to a conventional control and denote a process $(x, v)$.

For this problem, the cost function is the sum of two terms, both of which we want to keep small. The first is the average stock level deviation. The other is the sum of transaction charges for restocking; each intervention carries a flat rate charge "1," independent of the amount of new stock. There must be no excess stock at the end of the time period.

$\left(E_{0, x_{0}}\right)$ will be recognized as a special case of $\left(P_{0, x_{0}}\right)$ of Section I, in which $n=1$,

$$
L(x, u)=|x|, f(., ., .) \equiv-1, d(.) \equiv 1, \mathcal{D}=[0, \infty)
$$

and

$$
g(x)= \begin{cases}+\infty, & \text { if } x>0 \\ 0, & \text { if } x \leq 0\end{cases}
$$

Define the functions $V_{N}:[0, T] \times \Re \rightarrow \Re \cup\{+\infty\}, N=$ $0,1, \ldots$, as follows:

$$
V^{0}(t, x)= \begin{cases}+\infty, & \text { if } x>(T-t) \\ \frac{((T-t)-x)^{2}}{2}+\frac{x^{2}}{2}, & \text { if }(T-t) \geq x \geq 0 \\ \frac{(T-t)^{2}}{2}-x(T-t), & \text { if } 0>x\end{cases}
$$

and, for $N \geq 1$

$$
V^{N}(t, x)= \begin{cases}+\infty, & \text { if } x>(T-t) \\ N+\frac{((T-t)-x)^{2}}{2(2 N+1)}+\frac{x^{2}}{2}, & \text { if }(T-t) \geq x \geq 0 \\ N+\frac{((T-t)-x)^{2}}{2(2 N+1)}-\frac{x^{2}}{2}, & \text { if } 0>x \geq-\frac{(T-t)}{2 N} \\ N+\frac{(T-t)^{2}}{4 N}, & \text { if }-\frac{(T-t)}{2 N}>x .\end{cases}
$$

Now, define $V:[0, T] \times \Re \rightarrow \Re \cup\{+\infty\}$

$$
V(t, x)=\inf _{N \in\{0,1, \ldots\}} V^{N}(t, x) \quad \text { for }(t, x) \in[0, T] \times \Re \text {. }
$$

Proposition 4.1: (The Value Function): Take $V$ to be the function defined by (4.3). We have

$$
\inf \left(E_{t, x}\right)=V(t, x) \quad \forall(t, x) \in[0, T] \times \Re
$$

i.e., $V$ is the value function for $\left(E_{0, x_{0}}\right)$.

(An Optimal Policy) Fix $(t, x) \in[0, T] \times \Re^{n}$. There exists a nonnegative integer $N$ such that

$$
\inf \left(E_{t, x}\right)=V^{N}(t, x) .
$$

Let $\bar{N}$ be the smallest such integer.

If $\bar{N}=0$, then $v \equiv 0$ is an optimal impulse control (for $E_{0, x}$ ). If $\bar{N}>0$, then $x \leq(T-t)$ and

$$
v=\left\{t_{1}, \ldots, t_{\bar{N}}, \xi_{1}, \ldots, \xi_{\bar{N}}\right\}
$$

is an optimal impulse control, where $t_{1}, \ldots, t_{\bar{N}}, \xi_{1}, \ldots, \xi_{\bar{N}}$ are defined as follows.

A) $\quad-((T-t) / 2 \bar{N}) \leq x \leq(T-t)$. In this case

$$
\begin{aligned}
t_{j}= & \frac{2 \bar{N} x+(T-t)}{2 \bar{N}+1}+(j-1) \times \frac{2((T-t)-x)}{2 \bar{N}+1} \\
& \quad \text { or } j=1,2, \ldots, \bar{N} \\
\xi_{j}= & \frac{2((T-t)-x)}{2 \bar{N}+1} .
\end{aligned}
$$

B) $\quad x<-((T-t) / 2 \bar{N})$. In this case

$$
\begin{aligned}
t_{j} & =(j-1) \times \frac{(T-t)}{\bar{N}}, \quad \text { for } j=1,2, \ldots, \bar{N} \\
\xi_{1} & =\left(|x|+\frac{(T-t)}{2 \bar{N}}\right) \\
\xi_{j} & =\frac{(T-t)}{\bar{N}}, \quad \text { for } j=2,3, \ldots, \bar{N} .
\end{aligned}
$$

\section{Comments}

a) Minimizing processes are not unique. For $x_{0}>T$, it is not possible to satisfy the constraints of $\left(E_{0, x_{0}}\right)$. In this case, all processes have the same cost " $+\infty$ " and are, therefore, optimal, in a trivial sense. Even if $x_{0}<T$, for certain values of $T$ and $x_{0}$ the minimum cost can sometimes be achieved by jumping at time $t=0$ or by postponing the time of the first jump. The control described in Proposition 4.1 is the optimal control involving the least number of jumps. 
b) An analysis of the previous formulas for the $V^{N}$ 's provides the following characterization of $\bar{N}$, the minimum number of impulses of the optimal controls (for $\left(E_{t, x}\right)$ ). We have the following.

i) $\quad x>(T-t)$. In this case, $\bar{N}=0$.

ii) $0 \leq x \leq(T-t)$. In this case

$$
\begin{aligned}
\bar{N}=\min \{n \in\{0,1,2, \ldots\} \mid((T-t) & -x) \\
\leq & \sqrt{(3+2 n)(1+2 n)}\} .
\end{aligned}
$$

iii) $\quad x<0$. Define

$$
n_{1}= \begin{cases}\min \left\{n_{1}^{-}, n_{1}^{+}\right\}, & \text {if } 2 \leq \frac{(T-t)}{|x|} \\ +\infty, & \text { if } 2>\frac{(T-t)}{|x|}\end{cases}
$$

where

$$
\begin{aligned}
& \left.n_{1}^{-}=\max \left\{n \geq 1 \mid 2 n \leq \frac{(T-t)}{|x|}\right\}\right\} \\
& n_{1}^{+}=\min \{n \geq 1 \mid(T-t)-x \leq \sqrt{(3+2 n)(1+2 n)}\} \\
& \quad \text { and } \\
& n_{2}=\min \left\{n \geq 1 \mid(T-t) \leq 2 \sqrt{n(n+1)} \text { and } 2 n>\frac{(T-t)}{|x|}\right\} .
\end{aligned}
$$

Then, we can choose $\bar{N}$ to be the smallest integer in the set

$$
\arg \min \left\{n \rightarrow V_{n}(T-t, x) \mid n \in\left\{0, n_{1}, n_{2}\right\}\right\} .
$$

(Here, we interpret $V_{n}(T-t, x)=+\infty$ if $n=+\infty$.) An optimal state trajectory is illustrated in Fig. 1, for the case $T=9$ and $x_{0}=0$. Fig. 2 provides a plot of the value function.

Proof of the proposition is broken down into stages, the conclusions of each of which is summarized as a Lemma. First, we state, without proof, some properties of the $V^{N}$ 's, all of which are straightforward consequences of the defining relationships (4.1) and (4.2).

Define

$$
\mathcal{A}=\{(t, x) \in[0, T] \times \Re: x \leq T-t\} .
$$

Lemma 4.2: Fix $t \in[0, T]$ and an integer $N \geq 0$.

a) $\operatorname{dom}\left(V^{N}\right)=\mathcal{A} \cdot V^{N}(.,$.$) is the restriction to \mathcal{A}$ of a continuously differentiable function on $\Re \times \Re$.

b) The restriction of $V^{N}(t,$.$) to [0, \infty)$ is convex.

c) Define

$$
y(N, t):=\frac{T-t}{2(N+1)} .
$$

Then $V^{N}(t,$.$) is decreasing on (-\infty, y(N, t))$, increasing on $(y(N, t), \infty)$ and

$$
V^{N}(t, y(N, t))=N+\frac{(T-t)^{2}}{4(N+1)} .
$$

d) $V^{N}(t,$.$) is constant on (-\infty,-((T-t) / 2 N)$, if $N>0$. Lemma 4.3:

a) $V$ is a lower semicontinuous function, bounded from below, and $\operatorname{dom} V=\mathcal{A}$. The restriction of $V$ to $\mathcal{A}$ is locally Lipschitz continuous.

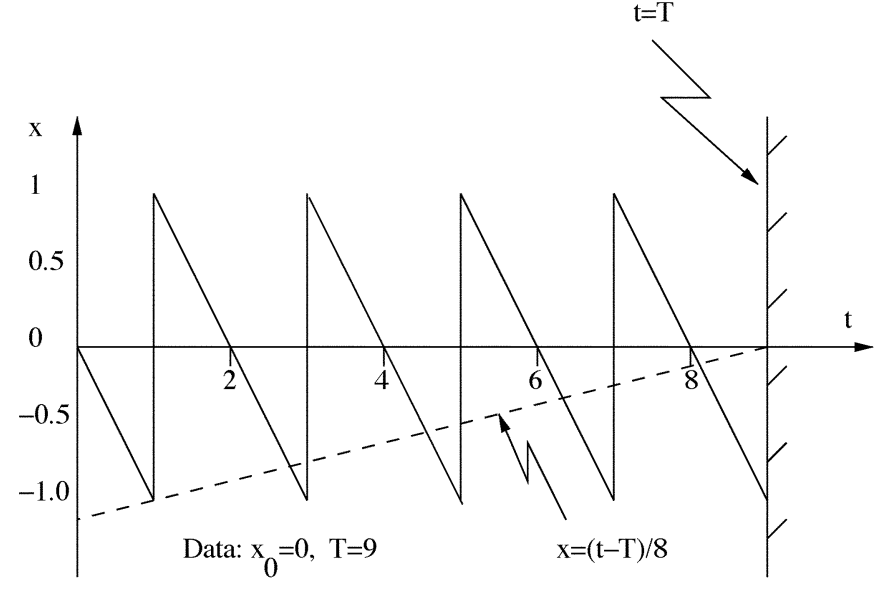

Fig. 1. Optimal state trajectory.

b) Take any bounded subset $D \subset \mathcal{A}$. Then, there exists a finite index set $J_{D} \subset\{0,1,2, \ldots\}$ with the property: corresponding to any point $(t, x) \in D$, there exists $N \in$ $J_{D}$ such that

$$
V(t, x)=V^{N}(t, x) .
$$

Furthermore, (4.4) implies $N \geq 1$, if $x<-(T-t)^{-1}-$ $((T-t) / 4)$.

c) $V(t,$.$) is decreasing on (-\infty, 0]$.

d) $V(T, x)=\Psi_{(-\infty, 0]}(x)$ for all $x \in \Re$.

Proof: The first part of b) is true because the $V^{N}$ 's are uniformly continuous on $D$ and

$$
\lim _{N \rightarrow \infty} \inf \left\{V^{N}(t, x):(t, x) \in[0, T] \times \Re^{n}\right\}=+\infty .
$$

Examining the formulas for $V^{0}$ and $V^{1}$ we see that, if $x<$ $-(T-t)^{-1}-((T-t) / 4)$, then $V^{1}(t, x)<V^{0}(t, x)$. It follows that $V(t, x) \leq V^{1}(t, x)<V^{0}(t, x)$. So, we must have $N \geq 1$; b) is proved. Property a) follows from b) and Lemma 4.2 a). c) is true because $V(t,$.$) inherits the monotonicity properties of the$ $V^{N}(t,$.$) 's on (-\infty, 0]$. d) follows from (4.1) and (4.2).

Lemma 4.4: Take $t \in[0, T)$ and $x, y \in \Re$ such that $x \leq y$. Then

$$
V(t, x) \leq V(t, y)+1
$$

Proof: We can assume that $y \leq T-t$ since, otherwise, $V(t, y)=+\infty$ and the assertion is automatically true. Suppose then that $x \leq y \leq(T-t)$.

A) $0 \leq x \leq T-t$. In this case, in view of Lemma 4.3b), there exists an integer $N \geq 0$ such that $V(t, y)=$ $V^{N}(t, y)$. We have

$$
\begin{aligned}
V(t, x)-V(t, y) \leq & V^{N+1}(t, x)-V^{N}(t, y) \\
\leq & \max \left\{V^{N+1}(t, 0)-V^{N}(t, y),\right. \\
& \left.V^{N+1}(t, y)-V^{N}(t, y)\right\} .
\end{aligned}
$$

In the second line, we have used the fact that $V^{N+1}(t,$.$) is convex on [0, y]$ [Lemma $\left.\left.4.2 \mathrm{~b}\right)\right]$ and therefore achieves its maximum at an extreme point of 


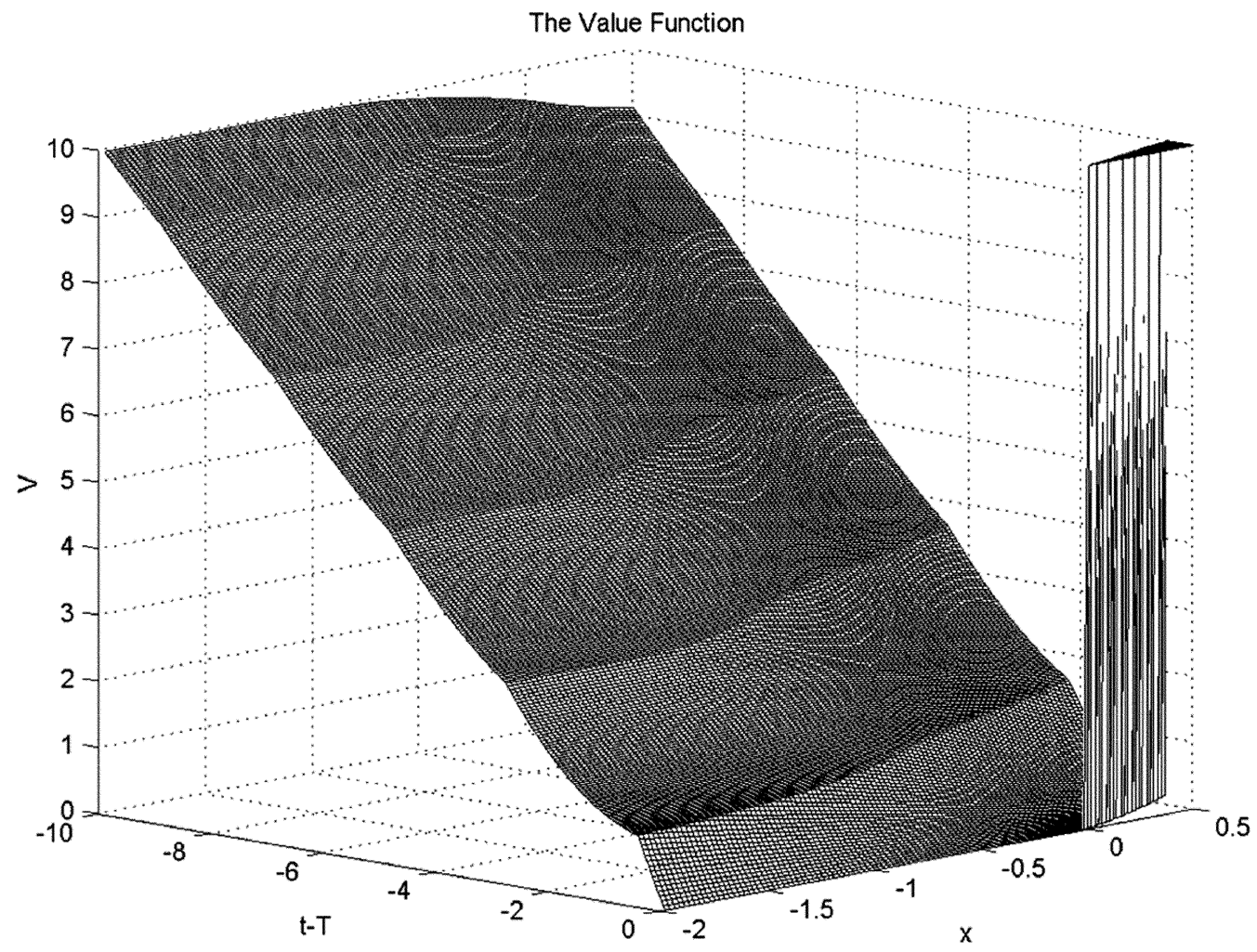

Fig. 2. Value function.

the interval $[0, y]$, namely 0 or $y$. However, by Lemma $4.2 \mathrm{c})$

$$
\begin{aligned}
V^{N+1}(t, 0)-V^{N}(t, y) \leq & N+1+\frac{(T-t)^{2}}{2(2 N+3)} \\
& -\min _{0 \leq y^{\prime} \leq T-t} V^{N}(t, y) \\
= & N+1+\frac{(T-t)^{2}}{2(2 N+3)} \\
& -N-\frac{(T-t)^{2}}{4 N+4}<1 .
\end{aligned}
$$

Also

$$
\begin{aligned}
V^{N+1}(t, y)-V^{N}(t, y)=N+1 & +\frac{|(T-t)-y|^{2}}{4 N+6} \\
& -N-\frac{|(T-t)-y|^{2}}{4 N+4}<1 .
\end{aligned}
$$

It follows that $V(t, x)-V(t, y) \leq 1$.

B) $\quad x<0$. In this case, set $x^{\prime}=\min \{-((T-t) / 2),-(T-$ $\left.t)^{-1}-((T-t) / 4)\right\}$. By Lemma $\left.4.2 \mathrm{~d}\right)$ and Lemma 4.3 b) and c)

$$
\begin{aligned}
V(t, x) & \leq V\left(t, x^{\prime}\right) \quad \text { and } \\
V\left(t, x^{\prime}\right) & =V^{N}\left(t, x^{\prime}\right) \quad \text { for some } N \geq 1 .
\end{aligned}
$$

Since $x^{\prime} \leq-((T-t) / 2)$ and $N$ is "minimizing"

$$
\begin{aligned}
V^{N}\left(t, x^{\prime}\right) & -V^{N^{\prime}}\left(t, x^{\prime}\right)=N+\frac{(T-t)^{2}}{4 N} \\
& -N^{\prime}-\frac{(T-t)^{2}}{4 N^{\prime}} \leq 0 \text { for all } N^{\prime} \geq 1 .
\end{aligned}
$$

Let $M \geq 0$ be an integer such that $V(t, y)=$ $V^{M}(t, y)$. Then, by Lemma $\left.4.2 \mathrm{c}\right)$,

$$
\begin{aligned}
V(t, y) & \geq V^{M}(t, y(t, M)) \\
& =M+\frac{(T-t)^{2}}{4(M+1)} .
\end{aligned}
$$

The proof is completed by noting that, in view (4.5), we can now deduce that

$$
\begin{aligned}
V(t, x)-V(t, y) & \leq V^{N}\left(t, x^{\prime}\right)-V^{M}(t, y(t, M)) \\
& =N+\frac{(T-t)^{2}}{4 N}-M-\frac{(T-t)^{2}}{4(M+1)} \\
& =N+\frac{(T-t)^{2}}{4 N}-(M+1)-\frac{(T-t)^{2}}{4(M+1)}+1
\end{aligned}
$$$$
\leq 1 \text {. }
$$

Lemma 4.5: For each $(t, x) \in(0, T) \times \Re$

$$
\xi_{0}-\mathcal{H}\left(x,-\xi_{1}\right) \geq 0, \quad\left(\xi_{0}, \xi_{1}\right) \in \partial^{P} V(t, x) .
$$

Proof: Take any $(t, x) \in(0, T) \times \Re$. We can assume that $(t, x) \in \mathcal{A}$, i.e., $x \leq T-t$, since, otherwise, $V(t, x)=+\infty$ and there is nothing to prove. We know $V(t, x)=V^{N}(t, x)$ for some $N \geq 0$. Making use of the formulas for $V^{N}$, we can show, by means of simple calculations, that

$$
\frac{\partial}{\partial t} V^{N}(t, x)-\frac{\partial}{\partial x} V^{N}(t, x)+|x| \geq 0 .
$$

(If $(t, x)$ is a boundary point of $\mathcal{A}$, the derivatives in the formula are taken to be limits of values at neighboring interior points.) 
Take any $\left(\xi_{0}, \xi_{1}\right) \in \partial V(t, x)$. Then there exists $M>0$ and $\epsilon>0$ such that

$$
\begin{aligned}
V\left(t^{\prime}, x^{\prime}\right)-V(t, x) & \geq\left(\xi_{0}, \xi_{1}\right) \\
& \cdot\left(\left(t^{\prime}, x^{\prime}\right)-(t, x)\right)-M\left|\left(t^{\prime}, x^{\prime}\right)-(t, x)\right|^{2}
\end{aligned}
$$

for all $\left(t^{\prime}, x^{\prime}\right) \in(t, x)+\epsilon \mathcal{B}$. Since $V(t, x)=V^{N}(t, x)$ and $V^{N}\left(t^{\prime}, x^{\prime}\right) \geq V\left(t^{\prime}, x^{\prime}\right)$

$$
\begin{aligned}
& V^{N}\left(t^{\prime}, x^{\prime}\right)-V^{N}(t, x) \geq\left(\xi_{0}, \xi_{1}\right) \\
& \cdot\left(\left(t^{\prime}, x^{\prime}\right)-(t, x)\right)-M\left|\left(t^{\prime}, x^{\prime}\right)-(t, x)\right|^{2}
\end{aligned}
$$

for all $\left(t^{\prime}, x^{\prime}\right) \in(t, x)+\epsilon \mathcal{B}$. Since $V^{N}$ is the restriction of a continuously differentiable function to $\mathcal{A}$, and, in the event $(t, x)$ is a boundary point of $\mathcal{A}$, the normal cone to $\mathcal{A}$ at $(t, x)$ is $\{\alpha(1,1): \alpha \geq 0\}$, we deduce that

$$
\left(\xi_{0}, \xi_{1}\right)=\left(\frac{\partial V^{N}}{\partial t}, \frac{\partial V^{N}}{\partial x}\right)+\alpha(1,1)
$$

for some $\alpha \geq 0$. It follows from Lemma 5.5 a) and b) that

$$
\begin{aligned}
\xi_{0}-\mathcal{H}\left(x,-\xi_{1}\right)\left(=\frac{\partial V^{N}}{\partial t}(t, x)\right. & +\alpha \\
& \left.-\frac{\partial V^{N}}{\partial x}(t, x)-\alpha+|x|\right) \geq 0 .
\end{aligned}
$$

Proof of Prop. 4.1: First, we show that the process $(x, v)$ of Proposition 4.1 is a minimizer. Here, we make use of the sufficient condition of optimality provided by Proposition 2.3, all hypotheses for the application of which are satisfied by the data for problem $E_{0, x_{0}}$.

Identify the function $\phi$ of Proposition 2.3 with $V$, defined by (4.3). Lemmas 4.5 and 4.4 establish that $\phi$ satisfies hypotheses $\left(\mathrm{a}^{\prime}\right)$ and $\left(\mathrm{b}^{\prime}\right)$ of Proposition 2.3.

Lemma 4.3 d) tells us that $V(T,)=.g($.$) . Since, however, the$ restriction of $V$ to $\operatorname{dom} V(=\{(t, x): x \leq T-t\})$ is continuous, we have

$$
\lim _{t^{\prime} \uparrow T, x^{\prime} \rightarrow x} V\left(t^{\prime}, x^{\prime}\right)=V(T, x)=g(x), \quad \forall x \in \Re .
$$

We see that hypothesis $\left(\mathrm{d}^{\prime}\right)$ of Prop. 2.3 is also satisfied.

Let $N$ be a nonnegative integer such that $V\left(0, x_{0}\right)=$ $V^{N}\left(0, x_{0}\right)$. Simple calculations, treating each of the different cases, " $x_{0}>T$," " $T>x_{0}>-(T) / 2 N$." and " $-T / 2 N>x_{0}$ " reveal that

$$
V\left(0, x_{0}\right)=V^{N}\left(0, x_{0}\right)=\int_{0}^{T}|x(t)| d t+g(x(T))+N(v) .
$$

However, this is (2.6) of Proposition 2.3. It follows from Proposition 2.3 that $(x, v)$ is a minimizer and $V\left(0, x_{0}\right)$ is the minimum cost for $\left(E_{0, x_{0}}\right)$.

It remains to note that, for any $(t, x) \in[0, T] \times \Re$, the preceding analysis with $T$ and $x_{0}$ replaced by $T-t$ and $x$ respectively, establishes that $V(t, x)$ is the minimum cost for $\left(E_{t, x}\right)$. We have shown that $V$ is the value function for $\left(E_{0, x_{0}}\right)$.

\section{APPENDIX}

ProOFs of TheOREM 2.2, Proposition 2.3, AND THEOREM 3.2

We state without proof the following proposition, concerning existence of optimal control policies and also properties of the value function. The first assertion follows from standard compactness arguments used in (impulse free) optimal control theory, applied to processes restricted to subintervals on which they are impulse free. Proof of the second assertion makes use of the monotonicity properties of the value function evaluated, on the one hand, along an arbitrary process and, on the other, along a minimizing process, expressed in infinitesimal form.

Proposition 4.6: Let $V:[0, T] \times \Re^{n} \rightarrow \Re \cup\{+\infty\}$ be the value function for $\left(P_{0, x_{0}}\right)$.

i) $V$ is lower semicontinuous and, for each $(t, x) \in[0, T] \times$ $\Re^{n},\left(P_{t, x}\right)$ has an optimal control policy.

ii) $V$ is a lower semicontinuous solution to QVI.

We also state, without proof, the following lemma, concerning, first, the monotonicity properties of solutions to QVI along arbitrary impulse free processes and, second, the existence of an optimal impulse free process, in relation to which the inequality in the above monotonicity properties is reversed. The analysis, based on viability theory, is along the same lines as that in [12] or [13, Ch. 12].

Lemma 4.7: Take a lower semicontinuous solution $\phi:[0, T] \times \Re^{n} \rightarrow \Re \cup\{+\infty\}$ of QVI. Then, the following hold.

a) For any interval $\left[t^{-}, t^{+}\right] \subset[0, T]$ and any impulse-free process $(x, u)$ on $\left[t^{-}, t^{+}\right]$we have

$$
\phi\left(t^{+}, x\left(t^{+}\right)\right) \geq \phi\left(t^{-}, x\left(t^{-}\right)\right)-\int_{t^{-}}^{t^{+}} L(s, x(s), u(s)) d s .
$$

b) For any $\left(t^{-}, x^{-}\right) \in[0, T) \times \Re^{n}$ such that $R_{\phi}\left(t^{-}, x^{-}\right)<0$ there exists $t^{+} \in\left(t^{-}, T\right]$ and a process $(\bar{x}, \bar{u})$ on $\left[t^{-}, t^{+}\right]$ such that

$$
\begin{gathered}
R_{\phi}(t, \bar{x}(t))<0, \quad \forall t \in\left[t^{-}, t^{+}\right) \\
\phi\left(t^{+}, x\left(t^{+}\right)\right) \leq \phi\left(t^{-}, x\left(t^{-}\right)\right)-\int_{t^{-}}^{t^{+}} L(s, x(s), u(s)) d s
\end{gathered}
$$

and either $R_{\phi}\left(t^{+}, \bar{x}\left(t^{+}\right)\right)=0$ or $t^{+}=T$.

Proof of Theorem 2.2: We know that the value function $V$ is a lower semicontinuous solution to QVI. Suppose that $\phi$ : $[0, T] \times \Re^{n} \rightarrow \Re \cup\{+\infty\}$ is a lower semicontinuous solution to QVI. We must show that $\phi$ is the value function, i.e.,

$$
\phi(s, y)=\inf \left(P_{s, y}\right)
$$

for all $(s, y) \in[0, T] \times \Re^{n}$. We assume without loss of generality that $s=0$ and $y=x_{0}$. It suffices then to show the following.

i) Given any process $\left(y, u, v=\left(t_{1}, \ldots, t_{N}, \xi_{1}, \ldots\right.\right.$, $\left.\left.\xi_{N}\right)\right)$ for $\left(P_{0, x_{0}}\right)$, we have

$$
\phi\left(0, x_{0}\right) \leq \int_{0}^{T} L(t, y(t), u(t)) d t+g(y(T))+\sum_{i=1}^{N} d\left(\xi_{i}\right) .
$$

ii) There exists a process $\left(\bar{y}, \bar{u}, \bar{v}=\left(\bar{t}_{1}, \ldots, \bar{t}_{\bar{N}}, \bar{\xi}_{1}, \ldots\right.\right.$, $\left.\left.\bar{\xi}_{\bar{N}}\right)\right)$ for $\left(P_{0, x_{0}}\right)$ such that

$$
\phi\left(0, x_{0}\right) \geq \int_{0}^{T} L(t, \bar{y}(t), \bar{u}(t)) d t+g(\bar{y}(T))+\sum_{i=1}^{N} d\left(\bar{\xi}_{i}\right) .
$$


Indeed, these conditions combine to establish that $(\bar{y}, \bar{u}, \bar{v})$ is a minimizer with cost $\phi\left(0, x_{0}\right)$ and so $\phi\left(0, x_{0}\right)=V\left(0, x_{0}\right)$.

Consider $i$ ). If $g(y(T))=+\infty$ there is nothing to prove. So, assume that $g(y(T))<+\infty$. Let $\left\{\left[t_{i}^{-}, t_{i}^{+}\right],\left(x_{i}, u_{i}\right)\right\}_{i=0}^{N}$ be the multiprocess representation of $(y, u, v)$.

Since $\phi(T, x(T))=g(y(T))<+\infty$, we have, from Lemma 4.3 , that $\phi\left(t_{N}^{-}, x_{N}\left(t_{N}^{-}\right)\right)<+\infty$ and

$\phi\left(t_{N}^{-}, x_{i}\left(t_{i}^{-}\right)\right) \leq g\left(T, x_{N}(T)\right)+\int_{t_{N}^{-}}^{T} L\left(s, x_{N}(s), u_{N}(s)\right) d s$.

By condition b) of Def. 2.1 then

$$
\begin{aligned}
\phi\left(t_{N-1}^{+}, x_{i}\left(t_{N-1}^{+}\right)\right) \leq \phi\left(t_{N}^{-}, x_{N}\left(t_{N}^{-}\right)\right) \\
+d\left(x_{i}\left(t_{i}^{-}\right)-x_{i-1}\left(t_{i-1}^{+}\right)\right) .
\end{aligned}
$$

Proceeding in this way, we show that $\phi\left(t_{N-1}^{+}, x_{N-1}\left(t_{N-1}^{+}\right)\right)$, $\phi\left(t_{N-1}^{-}, x_{N-1}\left(t_{N-1}^{-}\right)\right), \ldots \phi\left(t_{0}^{+}, x_{0}\left(t_{0}^{+}\right)\right), \phi\left(t_{0}^{-}, x_{0}\left(t_{0}^{-}\right)\right)<$ $+\infty$

$$
\phi\left(t_{i}^{-}, x_{i}\left(t_{i}^{-}\right)\right) \leq \phi\left(t_{i}^{+}, x_{i}\left(t_{i}^{+}\right)\right)+\int_{t_{i}^{-}}^{t_{i}^{+}} L\left(s, x_{i}(s), u_{i}(s)\right) d s
$$

for $i=0, \ldots, N$ and

$$
\begin{aligned}
\phi\left(t_{i-1}^{+}, x_{i-1}\left(t_{i-1}^{+}\right)\right) \leq \phi\left(t_{i}^{-}, x_{i}\right. & \left.\left(t_{i}^{-}\right)\right) \\
& +d\left(x_{i}\left(t_{i}^{-}\right)-x_{i-1}\left(t_{i-1}^{+}\right)\right)
\end{aligned}
$$

for $i=1, \ldots, N$. Summing these inequalities, and recalling that $\left\{\left[t_{i}^{-}, t_{i}^{+}\right],\left(x_{i}, u_{i}\right)\right\}_{i=0}^{N}$ represents $(y, u, v=$ $\left.\left(t_{1}, \ldots, t_{N}, \xi_{1}, \ldots, \xi_{N}\right)\right)$ and $\left(t_{0}^{-}, x_{0}\left(t_{0}^{-}\right)=\left(0, x_{0}\right)\right.$, we obtain

$$
\phi\left(0, x_{0}\right) \leq \int_{0}^{T} L(t, y(t), u(t)) d t+g(y(T))+\sum_{i=1}^{N} d\left(\xi_{i}\right) .
$$

Consider ii). We can assume that $\phi\left(0, x_{0}\right)<+\infty$ since, otherwise, there is nothing to prove. We can then use the construction of Algorithm 3.1 to generate the multiprocess representation $\left\{\left[t_{i}^{-}, t_{i}^{+}\right],\left(x_{i}, u_{i}\right)\right\}_{i=0}^{N}$ of a process $\left(P_{0, x_{0}}\right)$ with the desired properties. Recall that Algorithm 3.1 generates sequences $\left\{\left[t_{i}^{-}, t_{i}^{+}\right]\right\}$and $\left\{\left(x_{i}, u_{i}\right)\right\}$ with the properties $\left(t_{0}^{-}, x_{0}^{-}\right)=\left(0, x_{0}\right)$, the $\left[t_{i}^{-}, t_{i}^{+}\right]$'s are contiguous, the $\phi\left(t_{i}^{-}, x_{i}\left(t_{i}^{-}\right)\right)^{\prime}$ 's and $\phi\left(t_{i}^{+}, x_{i}\left(t_{i}^{+}\right)\right)$'s are finite

$$
\phi\left(t_{i}^{-}, x_{i}\left(t_{i}^{-}\right)\right) \geq \phi\left(t_{i}^{+}, x_{i}\left(t_{i}^{+}\right)\right)+\int_{t_{i}^{-}}^{t_{i}^{+}} L\left(s, x_{i}(s), u_{i}(s)\right) d s
$$

and either

$$
\begin{aligned}
\phi\left(t_{i}^{+}, x_{i}\left(t_{i}^{+}\right)\right) & =\phi\left(t_{i+1}^{-}, x_{i+1}\left(t_{i+1}^{-}\right)\right) \\
+ & d\left(x_{i+1}\left(t_{i+1}^{-}\right)-x_{i}\left(t_{i}^{+}\right)\right)
\end{aligned}
$$

or

$$
t_{i}^{+}=T \text {. }
$$

The algorithm terminates, when, for some value of $i$ (we label $i=N)$, (4.10) is satisfied.

It follows from Lemma 4.3 that these sequences can be constructed. To ensure that the algorithm generates a process for $\left(P_{0, x_{0}}\right)$, it remains to show that it terminates. Suppose to the contrary that the algorithm generates infinite sequences. Then $t_{i}^{+}<T$ for $i=1,2, \ldots$ We deduce from (4.8) and (4.9) that, for $j=1,2, \ldots$

$$
\begin{aligned}
& \phi\left(t_{j}^{+}, x_{j}\left(t_{j}^{+}\right)\right) \\
& \leq \phi\left(0, x_{0}\right)-\sum_{i=0}^{j} \int_{t_{i}^{-}}^{t_{i}^{+}} L\left(s, x_{i}(s), u_{i}(s)\right) d s \\
& \quad-\sum_{i=1}^{j} d\left(x_{i}\left(t_{i}^{-}\right)-x_{i-1}\left(t_{i-1}^{+}\right)\right) .
\end{aligned}
$$

However, this is not possible, since $L$ is bounded below and values of $d$ are strictly bounded away from zero. We conclude that the algorithm terminates.

We have that (4.8) is satisfied for $i=0, \ldots, N,(4.9)$ is satisfied for $i=0, \ldots, N-1$ and (4.10) is satisfied for $i=N$. Bearing in mind that $\left\{\left[t_{i}^{-}, t_{i}^{+}\right],\left(x_{i}, u_{i}\right)\right\}$ is a multiprocess representation of a process $\left(\bar{y}, \bar{u}, \bar{v}=\left(\bar{t}_{1}, \ldots, \bar{t}_{\bar{N}}, \bar{\xi}_{1}, \ldots, \bar{\xi}_{\bar{N}}\right)\right)$ for $\left(P_{0, x_{0}}\right)$, where $\bar{\xi}_{i}=x_{i}\left(\bar{t}_{i}^{-}\right)-x_{i-1}\left(t_{i-1}^{+}\right)$, and $\phi(T,)=.g($.$) ,$ we conclude that

$$
\begin{aligned}
g(\bar{y}(T))=\phi((T, \bar{y}(T)) & \leq \phi\left(0, x_{0}\right) \\
& -\int_{0}^{T} L(s, \bar{y}(s), \bar{u}(s)) d s-\sum_{i=1}^{N} d\left(\bar{\xi}_{i}\right)
\end{aligned}
$$

and so $\phi\left(0, x_{0}\right) \geq J_{0, x_{0}}(\bar{u}, \bar{v})$.

Property (ii) has been confirmed and proof of Theorem 2.2 is concluded. As a by-product of the last part of the proof of Theorem 2.2, we also have a proof of Theorem 3.3.

Proof of Proposition 2.3: The proposition is assertion i) of the proof of Theorem 2.2. Scrutiny of the preceding analysis reveals however that assertion $\mathrm{i}$ ) is true merely under the reduced hypotheses of Proposition 2.3.

\section{REFERENCES}

[1] J. P. Aubin, Mathematical Methods of Game and Economic Theory. Amsterdam, The Netherlands: North-Holland, 1982.

[2] — Viability Theory. Boston, MA: Birkhäuser, 1991.

[3] J. P. Aubin, J. Lygeros, S. Sastry, and N. Seube, "Impulse differential inclusions: a viability approach to hybrid systems," IEEE Trans. Automat. Contr., vol. 47, pp. 2-20, Jan. 2002.

[4] J. P. Aubin et al., "Optimal impulse control problems and quasivariational inequalities thirty years later," in Optimal Control and Partial Differential Equations, J. Menaldi et al., Eds. Paris, France: IOS Press, 2001, pp. 311-324.

[5] G. Barles, "Deterministic impulse control problems," SIAM J. Control Optim., vol. 23, pp. 419-432, 1985.

[6] E. N. Barron and R. Jensen, "Semicontinuous viscosity solutions for Hamilton-Jacobi equations with convex Hamiltonians," Comm. Partial Diff. Eqns., vol. 15, pp. 1713-1742, 1990

[7] A. Bensoussan and J. L. Lions, Contrôle Impulsionnel et Inéquations Quasi-Variationelles. Paris, France: Bordas, 1982. 
[8] A. Bensoussan and J. L. Minaldi, "Hybrid control and dynamic programming," Dyna. Continuous Discrete Impulsive Syst., vol. 3, pp. 395-442, 1997.

[9] I. Capuzzo-Dolcetta and L. C. Evans, "Optimal switching for ordinary differential equations," SIAM J. Control Optim., vol. 22, pp. 143-161, 1984.

[10] F. H. Clarke, Y. S. Ledyaev, R. J. Stern, and P. R. Wolenski, "Nonsmooth analysis and control theory," in Graduate Texts in Mathematics. Berlin, Germany: Springer-Verlag, 1998, vol. 178.

[11] E. Cruck, "Target problems under state constraints for nonlinear controlled impulsive systems," Univ. Bretagne Occidentale, Brest, France, Internal Rep. 01-2001, 2001.

[12] H. Frankowska, "Lower semicontinuous solutions to the Hamilton-Jacobi equation," SIAM J. Control Optim., vol. 31, pp. 257-272, 1993.

[13] R. B. Vinter, Optimal Control. Boston, MA: Birkhäuser, 2000.

[14] P. R. Wolenski and Y. Zhuang, "Proximal analysis and the minimal time problem," SIAM J. Control Optim., vol. 36, pp. 1048-1078, 1998.

[15] J. Yong, "Systems governed by ordinary differential equations with continuous, switching and impulse controls," Appl. Math. Optim., vol. 20, pp. 223-235, 1989.

[16] A. Bensoussan and J. L. Lions, "Impulse control and quasivariational inequalities," in Trans. J. M. Cole. Paris, France: Gauthier-Villars, 1984.

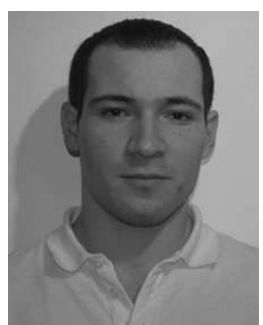

Daniel P. Berovic received the B.Sc. degree in engineering science from Oxford University, Oxford, U.K., in 1999, and the Ph.D. degree in the field of optimal hybrid control from Imperial College London, London, U.K., in 2003.

From 1999 to 2003, he was a Researcher with the Centre for Process Systems, Imperial College London, engaged in research in dynamic optimization, hybrid control, and process systems applications. He is currently employed by Mercer Investment Consulting, Birmingham, U.K.

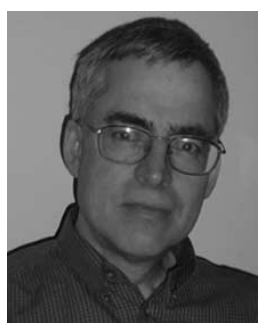

Richard B. Vinter (SM'88) received the B.Sc. degree in engineering science from Oxford University, Oxford, U.K., in 1969, and the Ph.D. degree in the field of optimal control from Cambridge University, Cambridge, U.K., in 1972.

$\mathrm{He}$ was a Postdoctoral Researcher (Harkness Fellowship) in the Electronic Systems Laboratory, Massachusetts Institute of Technology, Cambridge, MA, from 1972 to 1974. Since 1974, he has been with Imperial College London, London, U.K., where he is now Professor of Control Theory and Head of the Control and Power Group in the Electrical and Electronic Engineering Department. His research interests are in the areas of optimal control, differential games, hybrid control, and nonlinear estimation. 